\title{
Continuous spinal anaesthesia and analgesia for emergency caesarean section in a patient with severe kyphoscoliosis and acute lower respiratory tract infection
}

\author{
K Rupasingha $^{1 *}$, R Edirisinghe Pallemulle ${ }^{2}$ \\ Registrar ${ }^{1}$, Consultant Anaesthetist ${ }^{2}$, Department of Anaesthesiology, \\ Castle Street Hospital for Women, Colombo 8, Sri Lanka. \\ *Corresponding author: kishan_rupasingha@yahoo.com
}

\begin{abstract}
A primigravida with severe kyphoscoliosis and acute lower respiratory tract infection underwent emergency caesarean section (CS) under continuous spinal anaesthesia without complications in a major maternity hospital. This report highlights the anaesthetic challenges and the importance of early involvement of the anaesthesiologist.
\end{abstract}

Keywords: kyphoscoliosis in pregnancy; continuous spinal anaesthesia

\section{Case Report}

A 33 year old primigravida with severe kyphoscoliosis and gestational diabetes mellitus was admitted for elective CS at period of amenorrhoea of 38 weeks. On admission patient had a lower respiratory tract infection with fever for 2 days and oral antibiotics had been started. Patient was referred for anaesthetic assessment one day prior to surgery.

On examination patient was afebrile and mildly dyspnoeic, but not orthopnoeic. She was short, $139 \mathrm{~cm}$, weight was $40 \mathrm{~kg}$, and had gross kyphoscoliosis. She had a limping gait which was associated with congenital talipes. She was cardiovascularly stable and respiratory rate was around $24 / \mathrm{min}$ and on auscultation found to have a few rhonchi. Saturation on air was $93-94 \%$. Airway assessment showed a Mallampati III. Her metabolic equivalent was 4 . Lower lumbar interspinous spaces, especially L 3-4 was palpable.

\section{Investigations}

- WBC 16,000, Neutrophils $79 \%$, Haemoglobin 8.9 g/dl, Platelets 330,000, CRP $40 \mathrm{mg} / \mathrm{l}$

- $\quad$ ECG - Sinus rhythm, rate 76 bpm

- 2D-ECHO was normal
- Lung function tests

$\begin{array}{lcc}\text { Predicted } & \text { Best } & \text { \% Predicted } \\ \text { FVC } & 2.41 & 0.74 \\ 30 & & \\ \text { FEV1 } & 2.07 & 0.64 \\ 30 & & \\ \text { PEFR } & 5.55 & 1.64 \\ 29.5 & & \\ \text { FEV1/FVC } & 82.8 & 85.9 \\ 103 & & \end{array}$

Comment - Poor effort and cannot comment

Instructions were given to start chest physiotherapy.

Elective CS was planned for next day afternoon. Selected anaesthetic options at that stage were combined spinal epidural or continuous spinal anaesthesia. The mother was given explanations regarding the planned anaesthetic and reassured. A high dependency unit bed was reserved for post operative management. Ward staff was instructed to keep her fasting and to give acid aspiration prophylaxis.

On the day of the surgery patient had taken a heavy meal around $6 \mathrm{am}$. One hour later she became severely dyspnoeic and saturation dropped to $90 \%$ on air. She was propped up and nebulised with salbutamol and mask $\mathrm{O}_{2}$ was started. 
A couple of hours later the obstetric team noticed foetal heart irregularity with intermittent bradycardia. Therefore a decision was made to perform an emergency caesarean section, due to fetal and maternal compromise.

Instructions were then given for i.v. acid aspiration prophylaxis and the anaesthetic team prepared for an emergency CS under continuous spinal anaesthesia.

On arrival in the operating theatre she was still dyspnoeic and had occasional rhonchi. Blood pressure was $120 / 70 \mathrm{mmHg}, \mathrm{SpO}_{2} 97 \%$ on mask $\mathrm{O}_{2}$ and heart rate was 90-100/min. After establishing i.v. access, patient was positioned seated on the table with the legs hanging down over the side with feet on a stool. Dural puncture was done with an $18 \mathrm{G}$ epidural needle with the bevel facing downwards, attached to a $5 \mathrm{ml}$ syringe, using loss of resistance technique at the level of L3-L4 under aseptic conditions. Dural puncture was achieved on first attempt. Skin to subarachnoid length was $5 \mathrm{~cm}$ and an 18G epidural catheter was inserted with $2 \mathrm{~cm}$ kept inside directed caudally in the subarachnoid space. Catheter was sandwiched using sterile transparent dressings and fixed to her back and labelled as SA catheter.

$0.5 \%$ heavy bupivacaine $0.5 \mathrm{ml}$ with fentanyl $15 \mathrm{mcg}$ was injected while the mother was in sitting position. Sensory level was checked using ether after 5 minutes after lying down with a left lateral tilt. Sensory level to cold was T12. $0.5 \%$ heavy bupivacaine $0.5 \mathrm{ml}$ second bolus was given and catheter flushed with $1 \mathrm{ml}$ normal saline. Sensory level up to T8 level was achieved, and surgery commenced. Since patient complained discomfort another $0.5 \mathrm{ml}$ bupivacaine was given, and a sensory level of T5-6 was achieved. Blood pressure recorded at that time was $94 / 60 \mathrm{mmHg}$ which was corrected with intravenous fluids and release of caval compression.

Surgery was carried out without any complications and a baby girl was delivered. At the end of the surgery patient was transferred to HDU for monitoring and post operative pain relief. 90min after initial block, sensory level to cold was T12; and the patient was beginning to feel pain.
Continuous spinal plain bupivacaine $0.1 \%$ and fentanyl $2 \mathrm{mcg} / \mathrm{ml}$ infusion was commenced at a rate of $0.5 \mathrm{ml} /$ hour and she was comfortable with a pain score of $0-1$. She regained motor power while having the intrathecal infusion in HDU. Intravenous antibiotics were continued because of the chest infection. 2 litres $\mathrm{O}_{2}$ was continued postoperatively. At $6 \mathrm{pm}$ intrathecal infusion was stopped and she was transferred to the ward. The next morning patient was comfortable and spinal catheter was removed. She did not develop post dural puncture headache. (PDPH)

Post operative chest Xray and ultra sound scan of chest confirmed R/lower lobe consolidation. She was discharged home on oral antibiotics, with a healthy baby, on day 6 of hospital admission.
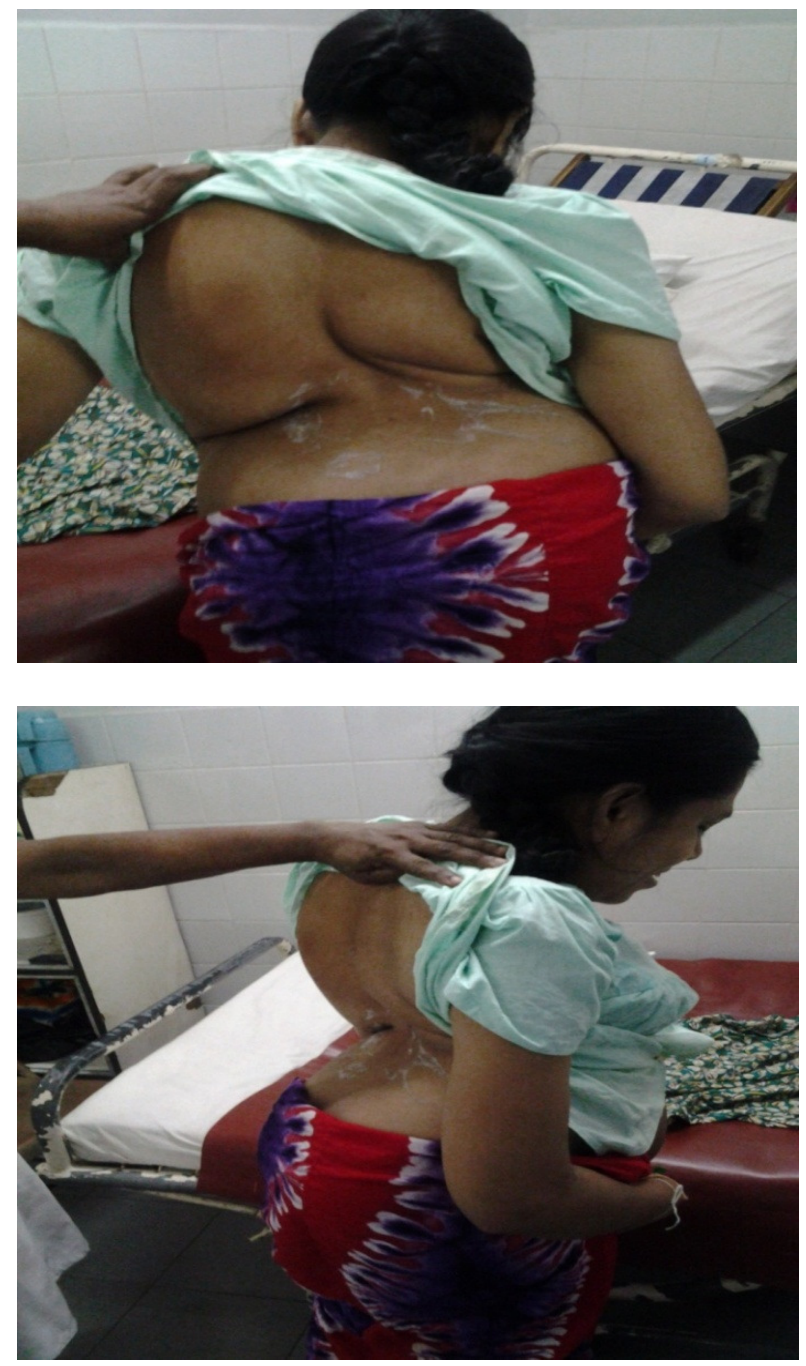

Pictures showing posterior and lateral curvatures 
Chest $\mathrm{X}$ ray showing tracheal deviation and right sided consolidation

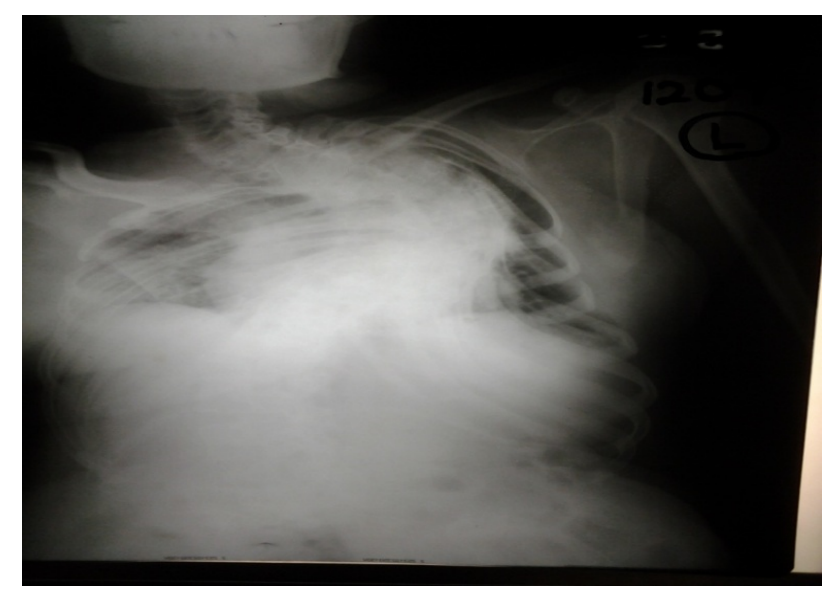

\section{Discussion}

Kyphoscoliosis is a complicated spinal deformity involving lateral and posterior curvature of the spine, vertebral body rotation and angulation of the ribs leading to thoracic rib cage deformity.

In kyphoscoliosis there is a fixation of the thoracic cage, and reduction in total lung capacities. Pregnancy further complicates this situation, as further reduction in the functional residual capacity with elevation of the diaphragm will not be neutralized by an increased inspiratory capacity. The net result in the pregnant kyphoscoliotic will be a further reduction in the vital capacity which was already reduced before pregnancy. ${ }^{1}$ Because of this they are more prone to get respiratory tract infections with secondary hypoxia affecting fetus as well. ${ }^{2}$ Our patient was admitted with a respiratory tract infection with low saturation on air. Rapid deterioration is anticipated when a chest infection is superimposed on chest deformity. The risks to the fetus are intra uterine growth restriction and pre term delivery.

Preoperative assessment should be focused on cardiovascular and respiratory systems. Any neurological deficits should be documented. ${ }^{2}$ Preoperative lung function testing is mandatory and showed severe restrictive disease in our patient.

The incidence of operative delivery is higher in these patients and neuraxial anaesthesia is considered as the technique of choice since general anaesthesia (GA) carries higher morbidity and mortality mainly due to restrictive lung disease, reduced lung reserve leading to ventilatory and weaning problems, and intubation difficulties in addition to other GA risks in normal pregnancy. This patient had very high risk of aspiration since she had taken a heavy meal $2 \mathrm{hrs}$ prior to the surgery.

Distortion of the spinous processes and rotation of the vertebral column makes it difficult to identify intervertebral spaces. ${ }^{3}$ Therefore central neuraxial blockade may be difficult to perform. Because of distorted spine, extension of local anaesthetic (LA) agents in subarachnoid and epidural space is unpredictable and may cause high spinal or inadequate or patchy blocks. It is also difficult to estimate the LA volume in single shot spinal with associated vertebral rotation and short stature as in our case. In addition to technical problems of performing a combined spinal epidural (CSE) anaesthesia, getting an adequate sensory level will be time consuming. In emergency surgery it is not practical as delivery of baby needs to be quick. Because of these problems we preferred continuous spinal anaesthesia even though it is not currently popular in obstetric anaesthetic practice. This technique allows controlled and gradual development of a dense regional block, while maintaining haemodynamic stability within a short period of time (10 -15 min).

Microcatheters (28-32 G) are no longer used in the developed countries because of the associated risk of cauda equina syndrome. ${ }^{4,5,6}$ Other factors which may contribute to cauda equina syndrome ${ }^{4}$ are using large volumes of heavy bupivacaine $(>3 \mathrm{cc})$ ${ }^{6}$ caudally placed catheters of $>3 \mathrm{~cm}$ and use of lignocaine. ${ }^{6}$

Most commercially available epidural kits including $17 \mathrm{G}$ or $18 \mathrm{G}$ Tuohy-type needle, can be used for dural puncture and placement of the catheter in the subarachnoid space. ${ }^{4}$ An advantage of using such catheters is the ease with which CSF can be withdrawn, confirming the correct placement of the catheter. The disadvantage is the frequent incidence of PDPH in the obstetric population after dural puncture with needles of this gauge. ${ }^{4}$ We used an $18 \mathrm{G}$ epidural needle with $18 \mathrm{G}$ catheter in our patient. Same size catheter as the needle is thought to reduce the CSF leak as it fits well in to the dural hole. ${ }^{6}$ We turned the bevelled end downwards which facilitated catheter 
advancement in caudal direction as a safety precaution to prevent high spread.

Heavy bupivacaine was selected as the drug of choice rather than plain bupivacain to minimize the high spread. Plain bupivacaine is the usual drug of choice in continuous spinal anaesthesia. ${ }^{6}$

The epidural catheter and filter has $1 \mathrm{ml}$ of dead space; therefore it is advised that a continuous spinal catheter be flushed with $1 \mathrm{ml}$ of saline after each bolus dose. ${ }^{4}$ However one must take care in doing this to avoid injecting large volumes of saline intrathecally.

Excellent post operative analgesia was achieved with subarachnoid infusion of LA and opioid combination. She was transferred to the ward early because of non availability of HDU/ICU bed.

Care must always be taken to clearly identify the spinal catheter to avoid the possibility of mistaking for an epidural catheter and sterile technique should always be used.

The timing of removal of the intrathecal catheter is a matter of controversy. It is advised to leave the catheter for a minimum period of 6-24 hours. ${ }^{7}$ The catheter should be left in place only if maintenance of sterility can be assured ${ }^{4}$.

\section{Conclusion}

Anaesthesia for a parturient with kyphoscoliosis is a challenge for an anaesthesiologist. The challenge worsens when other problems complicate the picture. Anaesthesiologist should be involved early in antenatal period for assessment, optimization and planning mode of anaesthesia for both elective and emergency surgery as well as for labour analgesia. The main anaesthetic options would be combined spinal epidural or continuous spinal anaesthesia.
Continuous spinal anesthesia can provide excellent surgical anesthesia for caesarean section in special situations where one has to overcome the problems of sudden cardio respiratory effects of single shot spinal. Eg: morbidly obese, complex cardiac disease and in situations where epidural spread of LA becomes highly unpredictable such as in severe kyphoscoliosis, previous spinal surgeries etc. Fear of PDPH and risk of spinal infections are the primary reasons for its infrequent use. However, the relative risk of these treatable side effects should be weighed against the many advantages of the technique in specific, challenging patient populations.

\section{References}

1. Sandler SW. Kyphoscoliosis and Pregnancy. South African Journal of Obstetrics and Gynaecology 1969; $13-15$.

2. Gupta S, Singaria G. Kyphoscoliosis and Pregnancy, Indian Journal of Anaesthesia 2004; 48:215-20.

3. Ko JY, Leffert LR. Clinical implications of neuraxial anaesthesia in parturient with scoliosis, Anaesthesia and analgesia 2009; 109:1930-4.

4. Creig M, Pulmar. Continuous spinal anaesthesia and analgesia in Obstetrics. Anaesthesia and Analgesia.2010;111(6):1476-9.

http://dx.doi.org/10.1213/ANE.0b013e3181f7e3f4 PMid:20889935

i. Hurley RJ, Lambert DH. Continuous spinal anesthesia with a microcatheter technique: preliminary experience. Anaesthesa and Analgesia 1990;70:97-102. http://dx.doi.org/10.1213/00000539-19900100000017

PMid:2242105

6. Burnell S, Byrne AJ. Continuous spinal anaesthesia. BJA CEPD Reviews 2001; 15: 134-7.

7. Clyburn P, Collis R. Oxford hand book of obstetric anaesthesia 1st edition 2008; 220 